\title{
Adaptive model reduction technique for large-scale dynamical systems with frequency-dependent damping
}

\author{
Xiang Xie (a,b,c,* Hui Zheng, $^{\mathrm{a}, * *}$, Stijn Jonckheere ${ }^{\mathrm{b}, \mathrm{c}}$, Axel van de Walle ${ }^{\mathrm{b}, \mathrm{c}}$, \\ Bert Pluymers ${ }^{\mathrm{b}, \mathrm{c}}$, Wim Desmet ${ }^{\mathrm{b}, \mathrm{c}}$ \\ ${ }^{a}$ State Key Laboratory of Mechanical System and Vibration, School of Mechanical \\ Engineering, Shanghai Jiao Tong University, Shanghai 200240, China. \\ ${ }^{b} K U$ Leuven, Department of Mechanical Engineering, Division PMA \\ Celestijnenlaan 300 - box 2420, B-3001 Leuven, Belgium. \\ ${ }^{c}$ Member of Flanders Make.
}

\begin{abstract}
In many engineering problems, it is customary to use viscoelastic materials in the passive control of structural vibration and noise radiation. The most commonly used numerical tool to obtain detailed information on the performance of complex structures is the finite element (FE) method. However, structures with viscoelastic materials often require very large-scale FE computational models to obtain reliable predictions, which makes original full-order system evaluation intractable due to memory and time limitations. In order to alleviate these problems, model order reduction techniques have become indispensable. Most of them, however, often do not handle complexvalued and especially frequency-dependent system matrices well. Due to the frequency dependency of the material properties, the FE equations of motion for viscoelastic models are not of a standard second-order form as that for regular elastic FE models. In this paper, a new transformation technique based on Taylor's theorem is introduced to treat the frequency-dependent shear modulus. After transforming the problem into a second-order system equation with remainder term, an adaptive second-order Arnoldi method is applied for the reduction of the computational complexity of structures, incorporating classical free- and constrained-layer damping treatments. In support, a relative error indicator is developed to iteratively enrich the re-
\end{abstract}

\footnotetext{
${ }^{*}$ Corresponding author: xiexiang@sjtu.edu.cn
}

**Corresponding author: huizheng@sjtu.edu.cn 
duced model and determine the position of expansion points used in next step. Three widely used models, the Golla-Hughes-McTavish model, Generalized Maxwell model and Fractional Derivative model for describing the frequency-dependent property of viscoelastic materials are investigated in order to demonstrate the simplicity, versatility and efficiency of the proposed approach.

Keywords: Viscoelastic material, Taylor's theorem, Adaptive model order reduction, Relative error indicator, Large-scale FE system

\section{Introduction}

Lightweight materials are frequently encountered in many engineering applications, such as aerospace, high-speed train, civil engineering, ship and submarine hulls, to reduce the weight and its associated energy consumption. However, the high stiffness-to-mass ratio, which is typical to these materials, can be often the cause of noise and vibration problems. In order to achieve the desired vibro-acoustic performance, the local or full-scale application of viscoelastic damping technologies to passively reduce the vibration levels by converting part of mechanical energy from a vibrating system into heat has become increasingly interesting [1]. Certainly, active and semi-active control methods can also be used to reduce the vibrations and noise in a dynamic system. However, due to their more complex and technical design, such as requiring electrical components or carefully calibrated sensors, active and semi-active control systems are usually not cost-effective solutions. Due to their microstructure, viscoelastic materials typically have a complex dynamic behavior which can be situated somewhere in between a solid and a viscous fluid. Moreover, its dynamic behaviour strongly depends on temperature and on frequency. Between both, an equivalence relation exists [2]. In general, three different types of behaviour can be observed. At low frequency, the material has a low stiffness and low damping whereas at higher frequencies, the material behaves more rigidly, still exhibiting (relatively) low damping. In between, in the so-called transition zone, the stiffness gradually evolves between the two asymptotes [3]. More importantly, the damping in this range is relatively large due to large relative displacements of the polymeric chains. The introduction of an accurate and efficient mathematical model used to include the frequency effect brings a lot of convenience in working with this class of materials [1]. According to the linear theory of viscoelastic- 
ity, the concept of complex modulus has gained extensive attention [4], and hence a number of mathematical models have been proposed to describe this complex modulus [4-10]. Its real part associates with the elastic property of the material, and defines the stiffness. Its imaginary part relates to the viscous property, and describes the energy dissipative ability of the material. Particularly, the Golla-Hughes-McTavish (GHM) [5, 6], Generalised Maxwell (GM) $[4,7]$ and Fractional Derivative (FD) [8-10] models are commonly used and all defined as a function of frequency. For a more detailed description of the various mathematical models of damping, the reader is referred to monograph [11] and review article [1].

In order to design products that meet the ever-increasing regulations and decrease the time-to-market, Computer Aided Engineering (CAE) has become essential. Among various CAE tools, an indispensable technique is the finite element method (FEM). Its use, however, often results in very large and time-consuming models especially in the presence of damping treatments. Evaluating frequency response functions is often necessary to observe the level of vibration and assess the influence of damping application. Hence, previously established models have to be inverted at each frequency of interests for calculating the response, which makes that system-level simulation is extremely prohibitive, even considering modern rapid developments in scientific supercomputing. As a result, there is a strong need for efficient computational approaches, capable of predicting the dynamic properties of systems with frequency-dependent viscoelastic damping at low cost.

Model order reduction (MOR) approaches have been developed to reduce the overall computational cost of numerical simulations while still maintaining a high degree of accuracy. The methods proposed in literature give satisfactory results for frequency-independent structures, but for systems with strongly frequency-dependent damping, numerical difficulties arise. The challenge in reducing frequency-dependent systems is that both the projection matrix and system matrices are frequency dependent, which is rather troublesome. Though extensive literature exists on reduction methods, indeed, few studies are dedicated to complex and frequency-dependent damping materials. Among the comparatively limited literature, the GM [12] and FD [13-15] damping models have received less attention compared to the GHM model [16-18]. The appearance of the complex material modulus represented by above-mentioned damping models exactly leads to the complex-valued and frequency-dependent global stiffness matrix, and further limits the applicability of MOR techniques. If one can figure out a way to solve the complex 
modulus, then most of reduced order methods can be directly applied to the viscoelastic structures without any difficulty. For example, the GHM method, by introducing auxiliary coordinates to account for the frequency-dependent modulus [16-18], adds viscoelastic properties to numerical models. Perhaps that is the reason why GHM model is widely studied by scholars in the model reduction of viscoelastic FE systems. However, such a method suffers from an increase in order of the final numerical model which is undesirable in many applications.

A key problem in MOR is to identify a suitable subspace to which the given problem can be restricted. In this present work, a simple yet very effective technique based on Taylor's theorem is proposed to handle the complex modulus such that a second-order dynamical system equation with remainder term is obtained. Subsequently, we just use the second-order part which holds the same form and matrix properties as a FE model with constant properties to construct the projection subspace and further to exploit this subspace for the computation of a frequency-independent and orthonormal reduction basis. However, it should be noted that the remainder term part is kept rather than omitting it for the derivation of final reduced-order model $(\mathrm{ROM})$. This way the inherent frequency dependence of the system is preserved in the projection, and is therefore also present in the ROM. The most common approach to the model reduction of second-order linear dynamical system is to transform the problem into an equivalent first-order form and then apply a Krylov subspace-based method. Such an approach, however, endures two major disadvantages; the dimension of system matrices is doubled and, more importantly, the second-order structure of the original full-order model (FOM) and essential properties, such as stability and passivity, are not retained within the ROM. Recently, the Second-Order Arnoldi algorithm (hereinafter referred to as SOAR-algorithm) was proposed by Bai and $\mathrm{Su}[19,20]$ which directly uses second-order Krylov subspace as the projection subspace. Thus, this algorithm not only reduces the computational complexity of the numerical simulations, but also still preserves the important second-order structure and matrix properties (e.g., symmetry) of the underlying problem, which makes the physical interpretation of the reduced system is achievable. Its superior numerical accuracy has been demonstrated in the work of [21] and thus this algorithm for computing an orthonormal projection basis of the second-order Krylov subspace is adopted herein. To the best knowledge of the authors, it appears that the present processing strategy for complex material modulus as well as the SOAR-algorithm has 
not been developed for reducing the dimension of large-scale viscoelastic material systems.

After selecting the MOR approach, the next important steps are to specify the expansion point location and its related order of projection basis required for the convergence. Therefore, a knowledge of the error between a reduced model and the original large-scale model is crucial. In order to avoid solving the FOM, the true error is often characterized by approximated error measurements, some of which can provide rigorous error bounds [22], others can't $[15,23,24]$. Probably the simplest approach to estimate the true model error is to calculate the difference between two successive reduced models from the start of initial order [24]. Nevertheless, this error indicator frequently underestimates the true error for complex models or models with complex material property [25]. In this paper, an error indicator for estimating the frequency response error is developed for practical engineering application of the SOAR-algorithm. Although no rigorous error bound and theoretical justification can be derived, we demonstrate that the present indicator is a good way to obtain reliable solution with an acceptable CPU time. Another advantage is its quite simple and straightforward implementation. With this error indicator, one could employ a procedure that iteratively improves the ROM predictions to satisfy a prescribed tolerance value. To reveal the beneficial versatility of the proposed approach for the study of frequency-dependent materials, all the above-mentioned viscoelastic models are incorporated into finite element equations of motion of structures, considering both free-layer damping (FLD) treatment and constrained-layer damping (CLD) treatment.

The remainder of the paper is organized as follows. In section 2 , three viscoelastic damping models as well as finite element formulation of structures with viscoelastic material are introduced. In section 3 , we present a MOR technique based on SOAR-algorithm for solving the frequency-dependent equation of motion. In section 4, we propose a error indicator for adaptively enriching the ROM. Numerical examples and discussions are presented in section 5. Conclusion and future work are in section 6 .

\section{Viscoelastic material models}

For modeling and calculation convenience, mathematical damping models have been developed. The complex shear modulus is typically used to describe the frequency-dependent characteristics of viscoelastic materials. 
When evaluated in the frequency domain, the complex modulus can be expressed in the following form:

$$
\bar{G}(\omega)=G_{s}(\omega)+\mathrm{j} G_{l}(\omega),
$$

where $\mathrm{j}^{2}=-1$ is the imaginary unit, $\omega=2 \pi f$, in which $f$ is the frequency in $\mathrm{Hz} ; G_{s}(\omega)$ and $G_{l}(\omega)$ are respectively named the storage and loss moduli. The non-dimensional quantity relating the imaginary and the real component of $\bar{G}$ is the loss factor that represents the dissipation capacity of the material.

$$
\bar{\eta}(\omega)=\frac{G_{l}(\omega)}{G_{s}(\omega)} .
$$

In what follows, three types of detailed representations of material modulus are summarized.

\subsection{Golla-Hughes-McTavish model}

The so-called Golla-Hughes-McTavish (GHM) model introduced by GollaHughes [5] and McTavish [6] uses a series of mini-oscillator terms which are a series of spring-mass-dashpot units to represent the shear modulus in the Laplace-transform domain.

$$
\bar{G}(s)=G_{r}\left(1+\sum_{i=1}^{q} \alpha_{i} \frac{s^{2}+2 \zeta_{i} \omega_{i} s}{s^{2}+2 \zeta_{i} \omega_{i} s+\omega_{i}^{2}}\right),
$$

where the complex Laplace transform symbol is $s=\mathrm{j} \omega$ with $\omega \geq 0$. $G_{r}$ is the equilibrium value of the modulus (or named static modulus); $\left(\alpha_{i}, \zeta_{i}, \omega_{i}\right)$ are the positive parameters, in which $\alpha_{i}$ is participation coefficient for the $i$-th mini-oscillator, $\zeta_{i}$ and $\omega_{i}$ denote damping ratio and natural frequency of the $i$-th dissipation equation. The number of mini-oscillator terms $q$ could be adjusted to reflect the different frequency dependence for different viscoelastic materials.

\subsection{Generalized Maxwell model}

One of the most popular linear models in describing damping materials is the Generalized Maxwell (GM) model [26], in which arbitrary spring-dashpot Maxwell elements as well as a static spring are assembled in parallel. Its complex modulus is represented by the expression:

$$
\bar{G}(s)=G_{r}+\sum_{i=1}^{m} \frac{G_{i} s \tau_{i}}{1+s \tau_{i}},
$$


in which $G_{i}$ and $\tau_{i}$ are respectively the shear modulus and relaxation time of the $i$-th Maxwell element. $m$ is the total number of Maxwell elements.

\subsection{Fractional Derivative model}

The introduction of fractional order derivatives to describe the stressstrain relationship of linear solid materials has resulted in a more powerful tool to model the physical phenomena related to viscoelasticity when compared to the integer order derivatives, and thus a number of fractional derivative models have been developed [8-10, 27]. One of these models, characterized by four parameters, has proved to be well suited for predicting the dynamic behaviour of damping materials over a wide frequency range. According to the developments made in Refs. [8-10], the corresponding shear modulus can be written as follows:

$$
\bar{G}(s)=\frac{G_{0}+G_{\infty}(s \tau)^{\alpha}}{1+(s \tau)^{\alpha}}
$$

in which $G_{0}$ is the dynamic modulus at zero frequency, i.e. the static modulus of elasticity, $G_{\infty}$ is the high frequency ultimate value of the dynamic modulus. $\tau$ is the relaxation time. $0<\alpha<1$ is fractional-order time derivative relating stress and strain fields. If $\alpha=0$, a pure spring is obtained while $\alpha=1$ represents a pure dashpot.

\subsection{Finite element formulation}

For the sake of easy implementation in commercial FEM codes, a modeling methodology is presented that uses standard volume element for the viscoelastic material. The FE discretisation of the differential equations of a problem consisting of a vibrating elastic structure (with frequencyindependent elastic properties) and a viscoelastic damping material (with frequency-dependent elastic properties) results in the following expression:

$$
\left[\mathbf{K}_{e}+\bar{G}(s) \mathbf{K}_{v}+s^{2} \mathbf{M}\right] \mathbf{X}=\mathbf{F}
$$

where $\mathbf{K}_{e}$ is the stiffness matrix with respect to the elastic structure, and $\mathbf{K}_{v}$ is the stiffness matrix related to the viscoelastic substructure and evaluated for a unit shear modulus. $\mathbf{M}$ is the global mass matrix. Note that these three matrices are $N \times N$ constant matrices, in which $N$ is the dimension of the model. For many practical models, this easily exceeds $10^{4}$. $\mathbf{X}$ and $\mathbf{F}$ are respectively the vectors of displacements and external loads. $\bar{G}(s)$ 
is the complex modulus used to represent the characteristics of frequencydependency as mentioned above. The output of this system is given by

$$
\mathbf{y}=\mathbf{L}^{\mathrm{T}} \mathbf{X}
$$

Eq. (7) is built to attain the transfer behavior of the dynamical system. Usually, $\mathbf{L}$ is an output measurement vector to calculate any variable of interest from the FE solution vector (e.g. dissipated power, average vibration velocity, etc.). In this work, it is only used to pick a certain degree of freedom (DOF). For simplicity, assuming that viscoelastic system only has one input and one output terminal. In addition, structural damping of the elastic base structure can be inserted into the $\mathrm{FE}$ equation by replacing $\mathbf{K}_{e}$ with a complex one $\mathbf{K}_{e}=\mathbf{K}_{e}(1+\mathrm{j} \eta)$, where $\eta$ is the loss factor of the base structure. However, it should be emphasized that the damping induced by the viscoelastic layer is commonly much more important than that of the elastic structure, and thus the structural damping will be ignored in the rest of this paper without loss of generality.

\section{MOR via SOAR-algorithm}

In principle, Eq. (6) can be directly used for solving the steady-state harmonic responses of the full-order damped structures at each frequency. However, such a procedure can be impractical when dealing with large-scale finite element models. This fact motivates the use of MOR techniques, which aims at reducing the system dimensions (and the associated computation cost) while still maintaining satisfactory accuracy. For viscoelastic systems, application of most of the MOR approaches is delicate since the constructed projection basis for capturing the dynamic behaviour should be updated successively as frequency varies. The simple yet powerful strategy proposed in this paper to cope with this is the transformation of the complex modulus $\bar{G}$ in Eq. (6) into higher order polynomials. According to Taylor's theorem, we expand $\bar{G}$ at a given point $s_{0}$ :

$$
\bar{G}(s)=G\left(s_{0}\right)+G^{\prime}\left(s_{0}\right)\left(s-s_{0}\right)+\frac{G^{\prime \prime}\left(s_{0}\right)}{2}\left(s-s_{0}\right)^{2}+R_{2}(s),
$$

where $R_{2}(s)$ denotes remainder term. In general, $\lim _{s \rightarrow s_{0}} R_{2}(s)=0$. Note that $s_{0}$ can be an arbitrary value, but needs to be fixed. In order to comply with the actual physical meaning $(s=\mathrm{j} \omega)$, the choice of a purely imaginary 
number is preferred. Then, substituting Eq. (8) into Eq. (6), it can be easily derived that

$$
[\underbrace{\left(\mathbf{K}_{e}+G \mathbf{K}_{v}-G^{\prime} s_{0} \mathbf{K}_{v}+\frac{G^{\prime \prime} s_{0}^{2} \mathbf{K}_{v}}{2}\right)}_{\mathrm{i}}+\underbrace{\left(G^{\prime} \mathbf{K}_{v}-G^{\prime \prime} s_{0} \mathbf{K}_{v}\right)}_{\text {ii }} s+\underbrace{\left(\mathbf{M}+\frac{G^{\prime \prime} \mathbf{K}_{v}}{2}\right) s^{2}}_{\text {iii }}+R_{2}(s) \mathbf{K}_{v}] \mathbf{X}=\mathbf{F}
$$

It should be noted that the $s_{0}$ in parenthesis related to $G$ and its derivatives is omitted for the sake of brevity. In practice, we are often interested in the approximation of Eq. (9) around a selected expansion point $s_{0} \neq 0$. In this case, Eq. (9) can be rewritten in the form

$$
\left[\tilde{\mathbf{K}}+\left(s-s_{0}\right) \tilde{\mathbf{D}}+\left(s-s_{0}\right)^{2} \tilde{\mathbf{M}}+R_{2}(s) \mathbf{K}_{v}\right] \mathbf{X}=\mathbf{F} .
$$

By performing straightforward algebraic manipulation and comparing the coefficients of Eq. (9) and Eq. (10), the following terms can be easily obtained.

$$
\tilde{\mathbf{K}}=\mathbf{K}_{e}+G \mathbf{K}_{v}+s_{0}^{2} \mathbf{M}, \quad \tilde{\mathbf{D}}=2 s_{0} \mathbf{M}+G^{\prime} \mathbf{K}_{v}, \quad \tilde{\mathbf{M}}=\mathbf{M}+G^{\prime \prime} \mathbf{K}_{v} / 2 .
$$

Through application of Taylor's theorem to handle the complex shear modulus, the frequency-dependent equation of motion of the viscoelastic system in the frequency- or Laplace-domain is easily converted into a higherorder (theoretically, infinite order) linear dynamical system. Moreover, this technique is independent of the damping model used (e.g., GHM, GM and FD even others).

Reduction of the dimension of system of equations formulated in Eq. (10) comes down to the selection of a suitable subspace as the projection subspace on which sufficient information about the essential dynamics of this system should be contained. The most well-known method in the subspace projection research is based on the construction of Krylov subspaces. The infinite order dynamical system can be numerically truncated to a system of finite order $k$ with $k \geqslant 2$ and then the $k$ th-order Arnoldi procedure [28] can be used to generate an orthonormal basis over that $k$ th-order Krylov subspace $[28,29]$. In this work, instead of spanning a higher-order Krylov subspace, we construct an orthonormal matrix whose columns only span the second-order Krylov subspace. The memory saving template of SOAR-algorithm which was introduced by Bai and $\mathrm{Su}$, see [19] and references therein, is selected to do so. We will focus on the immediate application of this algorithm, without going into details on elaborate issues about derivation and proving processes concerning this algorithm and others. 
By means of the SOAR-algorithm, we can generate an orthonormal and frequency-independent reduction basis $\mathbf{Q}_{n}$ with $\mathbf{r}_{0} \neq 0$ :

$$
\operatorname{span}\left\{\mathbf{Q}_{n}\right\}=\mathcal{G}_{n}\left(\mathbf{A}, \mathbf{B} ; \mathbf{r}_{0}\right)=\operatorname{span}\left\{\mathbf{r}_{0}, \mathbf{r}_{1}, \mathbf{r}_{2}, \cdots, \mathbf{r}_{n-1}\right\}
$$

where

$$
\left\{\begin{array}{l}
\mathbf{r}_{1}=\mathbf{A} \mathbf{r}_{0}, \\
\mathbf{r}_{\iota}=\mathbf{A} \mathbf{r}_{\iota-1}+\mathbf{B r}_{\iota-2}, \quad \text { for } \iota \geq 2
\end{array}\right.
$$

and the sequence $\mathbf{r}_{0}, \mathbf{r}_{1}, \mathbf{r}_{2}, \cdots, \mathbf{r}_{n-1}$ is called a second-order Krylov sequence based on the matrices $\mathbf{A}=-\tilde{\mathbf{K}}^{-1} \tilde{\mathbf{D}}$ and $\mathbf{B}=-\tilde{\mathbf{K}}^{-1} \tilde{\mathbf{M}}$ and starting vector $\mathbf{r}_{0}=\tilde{\mathbf{K}}^{-1} \mathbf{F}$. Such a subspace $\mathcal{G}_{n}\left(\mathbf{A}, \mathbf{B} ; \mathbf{r}_{0}\right)$ is called an $n$th second-order Krylov subspace since the vector $\mathbf{r}_{\iota}$ is obtained by a linear homogeneous recurrence relation of degree $2[20] . \mathrm{Q}_{n}$ denotes the $N \times n$ matrix with $n$ number of column orthonormal vectors. In most cases, it holds that $n \ll N$. The processing time for calculating the inverse of matrix $\tilde{\mathbf{K}}$ needs to be accelerated. Since $\tilde{\mathbf{K}}$ generated from FEM is a sparse matrix, a factorized form of $\tilde{\mathbf{K}}$, such as an LU factorization, should be made available before the iterative solution of the orthonormal basis $\mathbf{Q}_{n}$ for computational efficiency. The subspace spanned by the nonzero columns of $\mathbf{Q}_{n}$ can be used as the projection subspace to define a reduced system about the selected expansion point $s_{0}$. Multiplying Eq. (10) with $\mathbf{Q}_{n}^{\mathrm{T}}$ from the left yields the reduced system of the same form as

$$
\left[\tilde{\mathbf{K}}_{n}+\left(s-s_{0}\right) \tilde{\mathbf{D}}_{n}+\left(s-s_{0}\right)^{2} \tilde{\mathbf{M}}_{n}+R_{2}(s) \mathbf{K}_{v n}\right] \mathbf{X}_{n}=\mathbf{F}_{n}
$$

where $\tilde{\mathbf{K}}_{n}=\mathbf{Q}_{n}^{\mathrm{T}} \tilde{\mathbf{K}} \mathbf{Q}_{n}, \tilde{\mathbf{D}}_{n}=\mathbf{Q}_{n}^{\mathrm{T}} \tilde{\mathbf{D}} \mathbf{Q}_{n}, \tilde{\mathbf{M}}_{n}=\mathbf{Q}_{n}^{\mathrm{T}} \tilde{\mathbf{M}} \mathbf{Q}_{n}, \mathbf{K}_{v n}=\mathbf{Q}_{n}^{\mathrm{T}} \mathbf{K}_{v} \mathbf{Q}_{n}$ and $\mathbf{F}_{n}=\mathbf{Q}_{n}^{\mathrm{T}} \mathbf{F}$. The fundamental idea behind the formula $\mathbf{X} \approx \mathbf{Q}_{n} \mathbf{X}_{n}$ can be regarded as to approximate the vector $\mathbf{X}$ of the original full system by another vector $\mathbf{X}_{n}$ constrained to stay in the subspace $\mathcal{G}_{n}$ spanned by $\mathbf{Q}_{n}$.

Furthermore, by simple algebraic manipulation, Eq. (14) can be expressed as:

$$
\left[\mathbf{K}_{e n}+\bar{G}(s) \mathbf{K}_{v n}+s^{2} \mathbf{M}_{n}\right] \mathbf{X}_{n}=\mathbf{F}_{n}
$$

where $\mathbf{K}_{e n}=\mathbf{Q}_{n}^{\mathrm{T}} \mathbf{K}_{e} \mathbf{Q}_{n}$ and $\mathbf{M}_{n}=\mathbf{Q}_{n}^{\mathrm{T}} \mathbf{M} \mathbf{Q}_{n}$. Obviously, the transformed matrix triplet $(\tilde{\mathbf{K}}, \tilde{\mathbf{D}}, \tilde{\mathbf{M}})$ is adopted to compute the orthonormal basis $\mathbf{Q}_{n}$ of the projection subspace $\mathcal{G}_{n}$, but the original matrix triplet, (i, ii, iii) in Eq. (9) is 
directly projected onto the same subspace $\mathcal{G}_{n}$. Moreover, although the equation of motion in Eq. (6) is not the standard second-order dynamic system due to the presence of viscoelastic treatments, the subspace spanned by $\mathbf{Q}_{n}$ can still be used as the projection subspace similarly to conventional secondorder problems with constant properties. Without the truncation of Eq. (10) from infinite order to finite order, the reduced-order system in Eq. (15) holds the same form as the original full-order system in Eq. (6), which means that the inherent frequency dependence of the system is preserved in the ROM.

The highly efficient calculation of the transfer function, which can be visualised to assess the effectiveness of the damping treatment, is our main concern. The original transfer function $H_{N}(s)$ describes the input-output behaviour of large-scale system in Eqs. (6) and (7) and can be denoted by:

$$
H_{N}(s)=\mathbf{L}^{\mathrm{T}}\left[\mathbf{K}_{e}+\bar{G}(s) \mathbf{K}_{v}+s^{2} \mathbf{M}\right]^{-1} \mathbf{F} .
$$

Similarly, the reduced order transfer function $H_{n}(s)$ is of the form

$$
H_{n}(s)=\mathbf{L}_{n}^{\mathrm{T}}\left[\mathbf{K}_{e n}+\bar{G}(s) \mathbf{K}_{v n}+s^{2} \mathbf{M}_{n}\right]^{-1} \mathbf{F}_{n},
$$

where $\mathbf{L}_{n}=\mathbf{Q}_{n}^{\mathrm{T}} \mathbf{L}$.

It should be noted that the above-proposed algorithm can deal with both single-input single-output (SISO) and multiple-input multiple-output (MIMO) systems without any limitation. Specifically, due to the considered SISO system in this work, the transfer functions in Eqs. (16) and (17) are respectively a scalar function.

So far, the basic concept of the SOAR-algorithm and its straightforward implementation for solving a large-scale viscoelastic system has been presented. Next, this method will be extended with an adaptive selection procedure for the expansion points and its associated order in the reduced system, based on a stable error estimator.

\section{Adaptive procedure}

In the previous section, a framework for the MOR of viscoelastic materials was presented. Since obtaining better accuracy and smaller model size is the objective in the reduction process, important open problems are how many expansion points should be selected and how large the projection basis for each expansion point should be in order to build the ROM, preferably in an adaptive manner. This requires information about the total reduction error, 
which should be obtained in a computationally inexpensive way. Recall from Section 3 that there are two main contributions in the overall reduction error. One is the approximation error of the frequency-dependence in generating the frequency-independent reduction basis, which originates from truncating the Taylor series for the construction of second-order Krylov subspace. The second error is the reduction error in the classical sense, which originates from projecting the full-order model onto a reduction basis.

Next, we will try to respond to aforementioned open problems. A straightforward true relative transfer function error is firstly defined as

$$
E(s)=\frac{\left|H_{N}(s)-H_{n}(s)\right|}{\left|H_{N}(s)\right|},
$$

where $H_{N}(s)$ and $H_{n}(s)$ correspond to the transfer functions of the original model and of the reduced-order models, as defined above.

Knowing the error exactly in Eq. (18) requires the computation of the original system, which makes computations very expensive in the case of the high dimension of the problem. In this paper we propose a practical strategy to evaluate the error between the FOM and the ROM computed via the SOAR-algorithm. According to the estimated reduction error, the algorithm automatically selects the parameters (the location of expansion points and its related order of projection basis) used in later step and then adaptive MOR procedure can be achieved.

Before we proceed to the detail of whole calculation procedure for viscoelastic systems, we present the following two relative errors:

$$
\hat{\varepsilon}_{r+p}(s)=\frac{\left|H_{r}(s)-H_{r+p}(s)\right|}{\left|H_{r+p}(s)\right|}
$$

and

$$
\hat{E}(s)=\frac{\left|H_{r+p}^{(1)}(s)-H_{r+p}^{(2)}(s)\right|}{\left|H_{r+p}^{(2)}(s)\right|},
$$

in which, $H(s)$ refers to the transfer function of ROM, the subscripts $r$ and $r+p$ denote the dimension of ROM, the superscript (1) and (2) represent two different sets of ROMs.

The entire automatic procedure is schematically outlined in Fig. 1. In what follows, the general idea behind this procedure is explicitly explained step by step. 


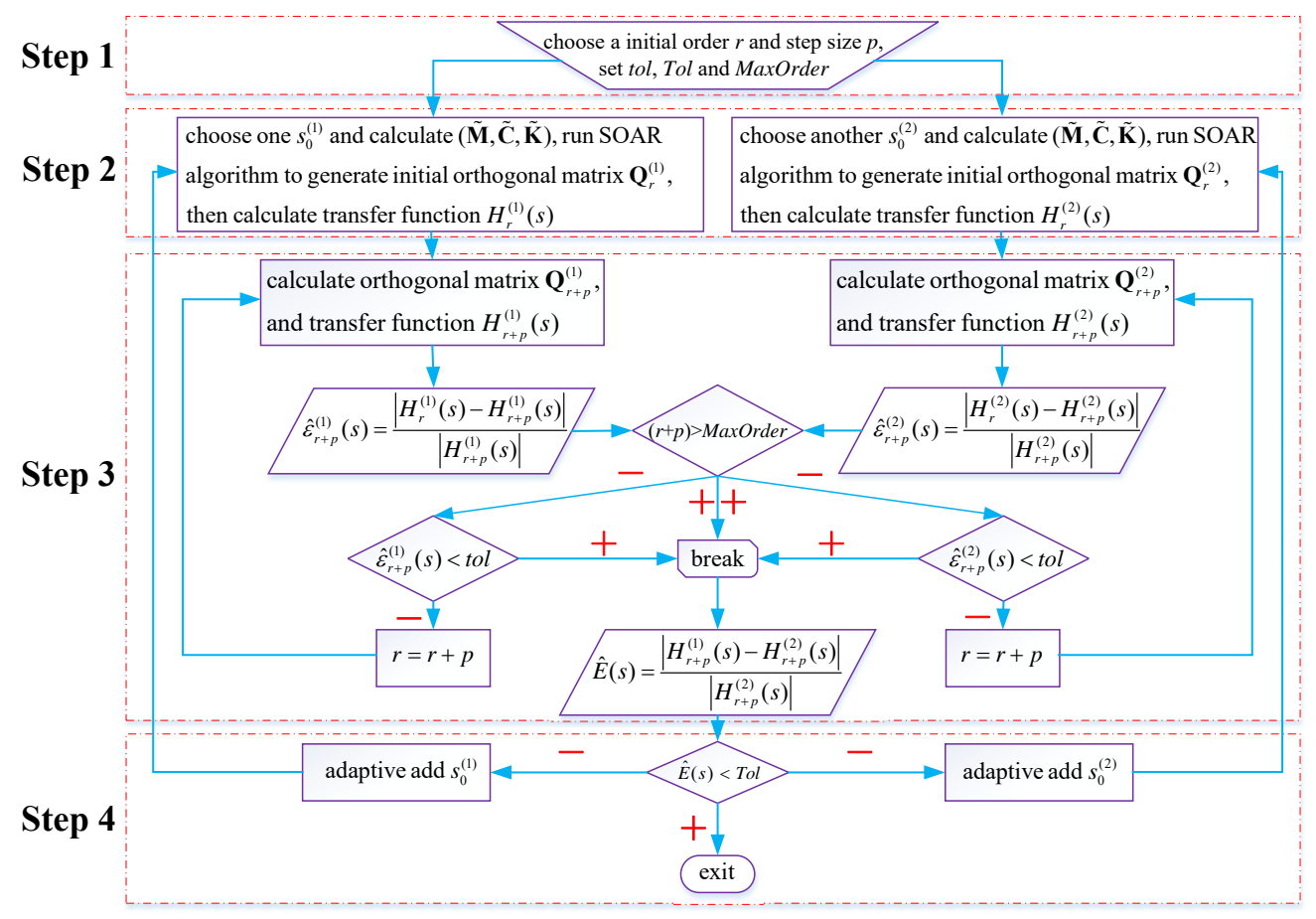

Figure 1: Adaptive enrichment strategy.

Step 1. Choose initial order $r$ and increased step size $p$. Set the prespecified termination tolerance tol for $\hat{\varepsilon}(s)$, Tol for $\hat{E}(s)$ and maximum order MaxOrder allowed for reduced model corresponding to each expansion point. Unless otherwise stated, the default value $T o l=1 \mathrm{e}-3$ is used as the final stopping criterion while the value of tol and MaxOrder are used as inner-loop stopping criteria. The considered frequency range is from $f_{\min }$ to $f_{\max }$, within which $f_{\text {mid }}=\left(f_{\min }+f_{\max }\right) / 2$ is defined.

Step 2. Select two unrelated expansion points $s_{0}^{(1)}$ and $s_{0}^{(2)}$ in the complex frequency plane $(s=\mathrm{j} \omega)$ that are symmetrically distributed around the middle of the complex frequency range of interest, i.e., $2 \pi \mathrm{j} f_{\text {mid }}$ (denoted by blue triangle), see Fig. 2, where the white square (red frame) corresponds to the first expansion point and the black circle (red rim) to the other. The use of complex expansion point typically results in a substantially smaller dimension of the ROM, since the expansion point can be put closer to the 


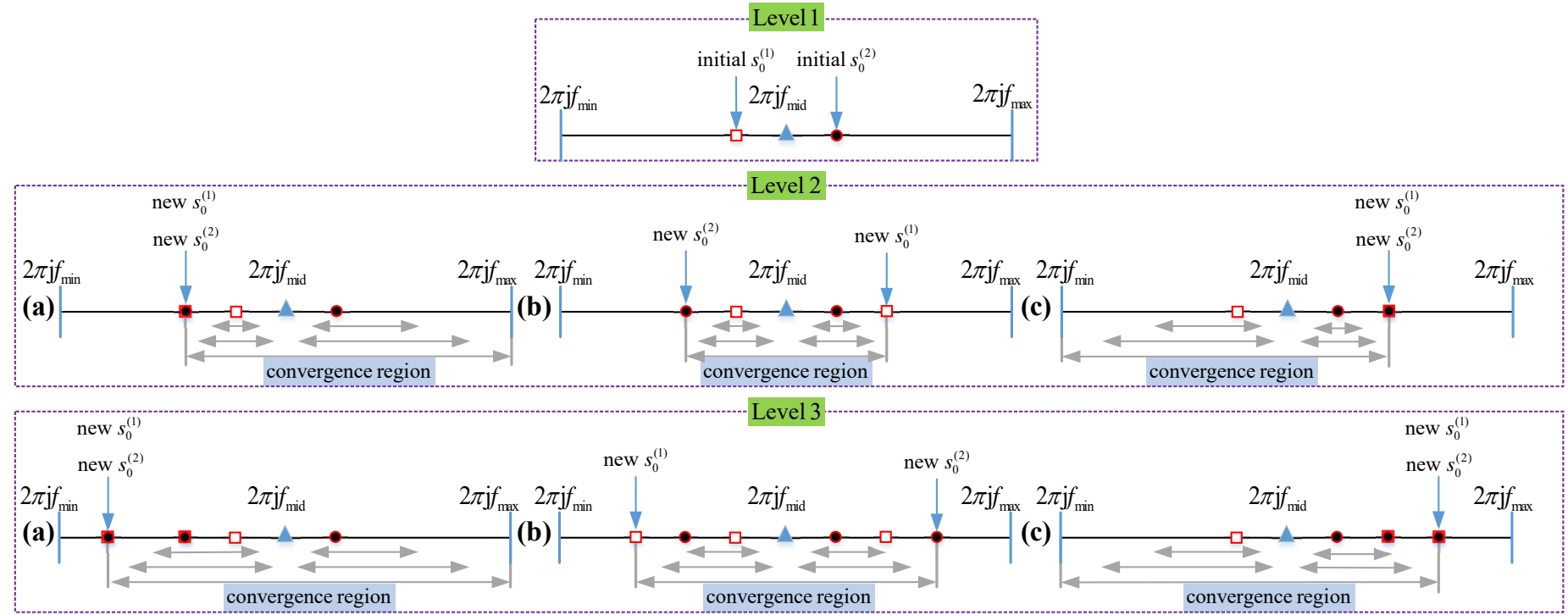

Figure 2: The algorithm for automatically selecting the expansion points.

frequency range of interest than any real expansion point. Choosing the positions near $2 \pi \mathrm{j} f_{\text {mid }}$ rather than the two ends of the considered frequency range as starting expansion points is more efficient since the local approximation property is mainly around the selected purely imaginary expansion points. Run the SOAR-algorithm to generate an $N \times r$ orthogonal projection matrix $\mathbf{Q}_{r}^{(1)}$ whose columns span an orthonormal basis of the second-order Krylov subspace $\mathcal{G}_{r}^{(1)}$. Compute $\mathbf{K}_{e r}^{(1)}, \mathbf{K}_{v r}^{(1)}, \mathbf{M}_{r}^{(1)}, \mathbf{F}_{r}^{(1)}$ and $\mathbf{L}_{r}^{(1)}$ to attain an approximant of the original transfer function, ie., $H_{r}^{(1)}$. In parallel, the SOAR-algorithm can be implemented to obtain the second transfer function $H_{r}^{(2)}$.

Step 3. Then gradually increase the order $r$ by every $p$ steps and run the SOAR-algorithm again to obtain the transfer function $H_{r+p}^{(1)}$. This procedure will break if the pre-specified termination tolerance of error is satisfied, i.e., $\hat{\varepsilon}_{r+p}^{(1)}<$ tol or if the maximum number of dimension allowed is reached, i.e., $(r+p)>$ MaxOrder. This means that there is no need to approximate the full system better by using more orthonormal vectors. Otherwise, repeat this step until one of the prescribed inner-loop stopping criteria is satisfied. Meanwhile, the SOAR-algorithm is applied to calculate the second transfer 
function $H_{r+p}^{(2)}$ with the order $r+p$. It should be noted that the final order $(r+p)$ for two distinct expansion points could be different. The transfer functions $H_{r+p}^{(1)}$ and $H_{r+p}^{(2)}$ are compared to calculate the relative error $\hat{E}(s)$ (i.e., to check each other's accuracy). If the final stopping criterion $\hat{E}(s)<$ Tol is fulfilled, exit. Then both ROMs are converged. Of course, ROM with smaller dimension in these two models is preferred to the other one for further application.

Step 4. If the final stopping criterion $\hat{E}(s)<$ Tol is not met, new expansion points have to be chosen in the region where the $\hat{E}(s)>\mathrm{Tol}$, which is generally far away from the previous expansion points. The cross point between the relative error $\hat{E}(s)$ and the threshold $T o l$ that is closest to $2 \pi \mathrm{j} f_{\text {mid }}$ is selected as an new expansion point. According to the convergence region, another expansion point can be determined based on three possible cases, as illustrated in Fig. 2. For the cases (a) and (c) of Level 2, the second $s_{0}$ is placed in the same position as the first $s_{0}$. As for the case (b), the second $s_{0}$ is placed in the another not yet converged region so that two interlaced sets of expansion points are produced. Each new expansion point is then separately added towards the two sets of expansion points of previous Level to perform the MOR procedure.

Iterate steps 3 and 4 again until the overall ROMs are satisfactory.

A few remarks are in order:

1. At step 1, usually, $1<p<N$ is a designated positive integer. Obviously, when $p=1$ and $(r+p) \rightarrow N$, the simplest relative transfer function error and true relative transfer function error as defined in Eq. (18) are realized. The advantages of choosing $p>1$ are twofold. On the one hand, sufficiently small error $\hat{\varepsilon}_{r+p}$ can be accomplished for $p=1$ even at some regions where the calculated transfer functions both are inaccurate, resulting in an error that does not reflect a reasonable estimate for the reduction error. This problem which is impossible to be completely solved, however, can be mitigated by simply adopting $p>1$. On the other hand, obtaining the system order $n$ of the final ROM desired to reach convergence can be sped up for the same initial order in terms of not evaluating the error at every iteration but only every $p$ steps.

2. At step 2, two different expansion points are sought, which leads to the construction of two distinct projection matrices and consequently 
the generation of two distinct ROMs. It should be admitted here that the reduction effort is doubled, as two sets of expansion points are calculated independently. However, this technique ensures that the resulting ROMs are accurate. This is a common trade-off between computational speed and accuracy.

3. At step 3, by gradually increasing orthonormal vectors to reduction basis, we aim to decrease the reduction error as mentioned before. Where proposed approximated relative error $\hat{E}(s)<t o l$, we assume that true relative error $E(s)<t o l$. That is to say, the true transfer function error is small at those frequencies where both ROMs are capable of approximating the full-order transfer function very well and have a low error estimation themselves.

4. At step 4, the moment-matching property indicates that the ROM using single complex expansion point can only capture the local characteristics within a certain frequency range near such expansion point, otherwise the ROM will become inaccurate beyond that frequency range [30]. Therefore, one may use more than one expansion points to reduce the approximation error of the frequency-dependence in generating the projection basis by enriching the information of subspaces. Since the procedure only selects new expansion points when this is really necessary, a very limited number of expansion points are required. This is done to make the computation of the reduction basis cheap in case of excessive and wasteful iterations. Also, adaptively selecting the new interlaced expansion points provides a fair opportunity for each of the two ROMs to converge over the whole desired frequency range. As multiple expansion points have their own projection basis, an orthogonalization procedure using the modified Gram-Schmidt or Singular Value Decomposition (SVD) should be applied to combine them into a union of reduction bases generated from multiple second-order Krylov subspaces. In this work, the orthonormal basis for the range of a matrix is obtained based on SVD.

In Ref. [31], Grimme pointed out that some error estimators may underestimate errors corresponding to sharp frequency peaks. In this paper, due to the presence of strongly frequency-dependent damping materials which attenuate the resonance peak, this problem is non-existent.

In conclusion, the present strategy uses an error indicator to successively refine two ROMs by increasing their order and expansion points in 
the Laplace space where the estimated error is larger than the predetermined threshold. By the way, although not presented here on account of the damping treatment, the proposed error indicator incorporated with SOARalgorithm can be directly implemented to the classical second-order dynamical systems.

\section{Numerical results and discussion}

In this section, we present three numerical examples to demonstrate the promising potential of the proposed solution procedure for solving structural vibration problems including viscoelastic materials with frequency-dependent property described by three different damping models. All our codes are implemented in MATLAB ${ }^{\circledR}$. All data about $\mathbf{K}_{e}, \mathbf{K}_{v}, \mathbf{M}, \mathbf{F}$ and $\mathbf{L}$ are extracted from COMSOL Multiphysics ${ }^{\circledR}$ with MATLAB ${ }^{\circledR}$. All numerical tests are performed on a Windows Machine with Intel Core(TM) i7-6560U CPU at $2.20 \mathrm{GHz}$ and $8 \mathrm{~GB}$ of RAM.

The frequency dependency of the complex shear modulus is described respectively by a GHM model with 5 mini-oscillator terms, a GM model with 12 Maxwell elements and a FD model with 4 arguments, whose parameters were identified through inverse characterisation from experimental data at a given temperature by Lima et al. [32], by Pirk et al. [33] and by Rouleau et al. [34], respectively and are tabulated in Tables 1 to 3 . The parameter values for GHM model have made minor modification so as to conform the considered frequency range. The storage modulus $G_{s}$ and loss factor $\bar{\eta}$ from the adopted models are shown in Fig. 3. The density concerning the viscoelastic layer is uniformly selected as $\rho=1400 \mathrm{~kg} / \mathrm{m}^{3}$ and the Poisson's ratio is assumed to be constant $\nu=0.495$. The modeling methodology presented here is to use classical shell elements for the elastic main structure and constrained-layer, and solid elements for the viscoelastic material. In COMSOL Multiphysics ${ }^{\circledR}$, a shell can be coupled to a solid by adding a Solid Connection node at the boundary level in the Shell interface and a Shell Connection node in the Solid Mechanics interface. The shell is modeled at a separate boundary and the connection distance between the shell and the face of the solid is taken into account in order to apply the correct shift of the shell's neutral line [35].

\subsection{Free-layer damping of a cantilever beam with GHM model}

The first example comprises the frequency response analysis of a viscoelastic system of order $N=17640$, which comes from a finite element 
Table 1: GHM model parameters [32].

\begin{tabular}{cccc}
\hline$i$ & $\alpha_{i}$ & $\omega_{i}$ & $\zeta_{i}$ \\
\hline 1 & 0.256 & 991.3320 & 4.575 \\
2 & 0.949 & 6986.160 & 4.270 \\
3 & 2.040 & 103437.5 & 2.702 \\
4 & 3.695 & 22950.07 & 1.923 \\
5 & 53.67 & 26646.56 & 1.299 \\
\hline \multicolumn{4}{l}{$G_{r}=0.28 \mathrm{MPa}$} \\
\hline
\end{tabular}

Table 2: GM model parameters [33].

\begin{tabular}{cccccc}
\hline$i$ & $G_{i}(\mathrm{MPa})$ & $\tau_{i}$ (second) & $i$ & $G_{i}(\mathrm{MPa})$ & $\tau_{i}$ (second) \\
\hline 1 & 0.041 & $3.90 \mathrm{e}-1$ & 7 & 6.852 & $5.68 \mathrm{e}-4$ \\
2 & 0.106 & $1.14 \mathrm{e}-1$ & 8 & 10.05 & $2.27 \mathrm{e}-4$ \\
3 & 0.225 & $5.27 \mathrm{e}-2$ & 9 & 12.69 & $8.84 \mathrm{e}-5$ \\
4 & 0.554 & $1.43 \mathrm{e}-2$ & 10 & 17.83 & $2.94 \mathrm{e}-5$ \\
5 & 1.065 & $5.00 \mathrm{e}-3$ & 11 & 15.48 & $5.27 \mathrm{e}-6$ \\
6 & 2.954 & $1.70 \mathrm{e}-3$ & 12 & 16.99 & $1.85 \mathrm{e}-6$ \\
\hline \multicolumn{6}{l}{$G_{r}=0.2 \mathrm{MPa}$} \\
\hline
\end{tabular}

Table 3: FD model parameters [34].

\begin{tabular}{cccc}
\hline$G_{0}(\mathrm{~Pa})$ & $G_{\infty}(\mathrm{Pa})$ & $\tau$ (second) & $\alpha$ \\
\hline $36.56 \mathrm{e} 3$ & $365.6 \mathrm{e} 6$ & $2.239 \mathrm{e}-6$ & 0.8 \\
\hline
\end{tabular}

model of an aluminum cantilever beam with free-layer damping treatment, as depicted in Fig. 4. The beam is clamped at one end and subjected to a normal unit point force at the other end. The displacement response at the free end of the shell is calculated. The dimensional length, width and thickness of each layer are respectively given by $0.3 \mathrm{~m} \times 0.025 \mathrm{~m} \times 2 \mathrm{~mm}$ and $0.3 \mathrm{~m} \times 0.025 \mathrm{~m} \times 1.5 \mathrm{~mm}$. The elastic aluminum structure is characterized by the following material properties: $E=70 \mathrm{GPa}, \rho=2700 \mathrm{~kg} / \mathrm{m}^{3}, \nu=0.3$. The material parameters of GHM model can be referred to Table 1 . The considered frequency range is from $f_{\min }=2 \mathrm{~Hz}$ to $f_{\max }=1000 \mathrm{~Hz}$ with a 2 $\mathrm{Hz}$ frequency step. Two expansion points $s_{0}^{(1)}=2 \pi \mathrm{j} * 400$ for the ROM 1 and $s_{0}^{(2)}=2 \pi \mathrm{j} * 600$ for the ROM 2 around $2 \pi \mathrm{j} f_{\text {mid }}=2 \pi \mathrm{j} * 500$ are selected to perform the SOAR-algorithm. The empirical values are taken to be: $r=p=5$, tol $=1 \mathrm{e}-2$ and MaxOrder $=400$. 

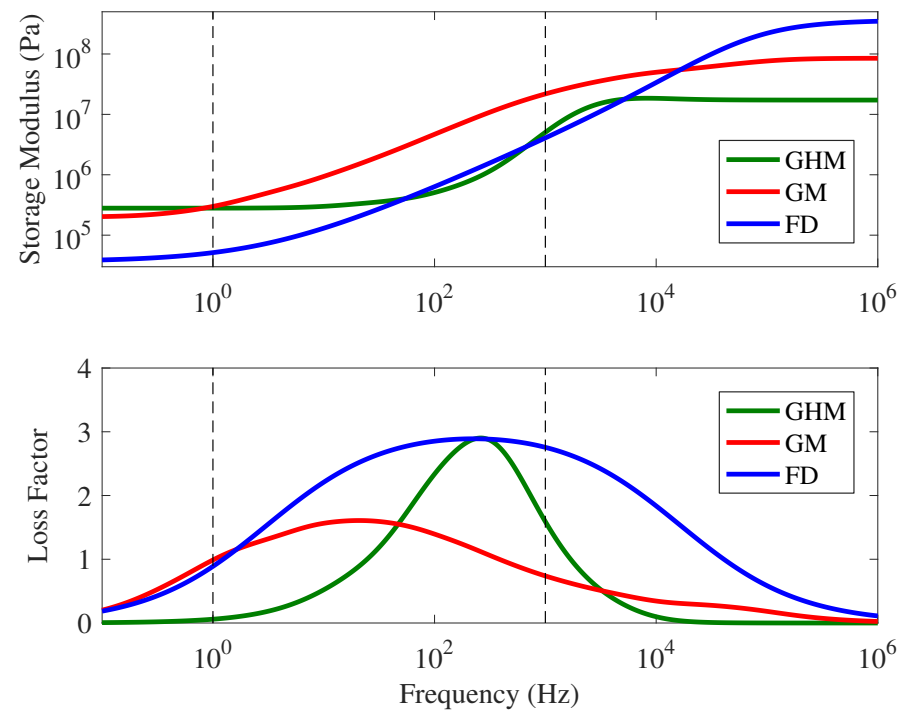

Figure 3: Viscoelastic frequency-dependent storage mudulus and loss factor.

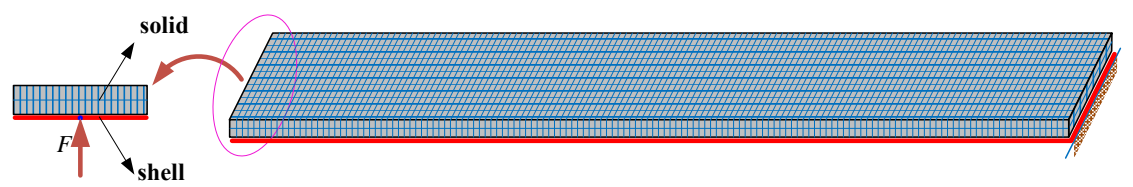

Figure 4: Schematic of the cantilever beam with free-layer damping.

Fig. 5 shows the resulting approximated transfer functions with the reduced order $n=15$ for the ROM 1 and with $n=15$ for the ROM 2, together with the exact transfer function $H_{N}(s)$ which serves as a reference. The true relative error for these two ROMs and the estimated error using the proposed error indicator are shown at the second plot of Fig. 5, in which the blue dashed line denotes the set tolerance of Tol. It is clear from Fig. 5 that the suggestion on the choice of purely imaginary expansion points offers good local approximation property around the chosen frequencies (such as $400 \mathrm{~Hz}$ and $600 \mathrm{~Hz}$ ). Note that for this case, no new expansion points need to be selected for the next Level calculation, which implies that efficient approximation of the exact transfer function can be achieved in one expansion point by a single-point moment-matching. It also implies that model reduction using second-order Krylov subspace instead of higher-order Krylov subspace 
is sufficient to yield desired accuracy. Furthermore, the estimated relative error almost follows the larger of the two true relative error curves, which means that once the estimated error has been reduced to satisfy a prescribed threshold, the two true relative error are naturally less than the threshold. That is to say, this result verifies that the assumption at step 3 is correct.
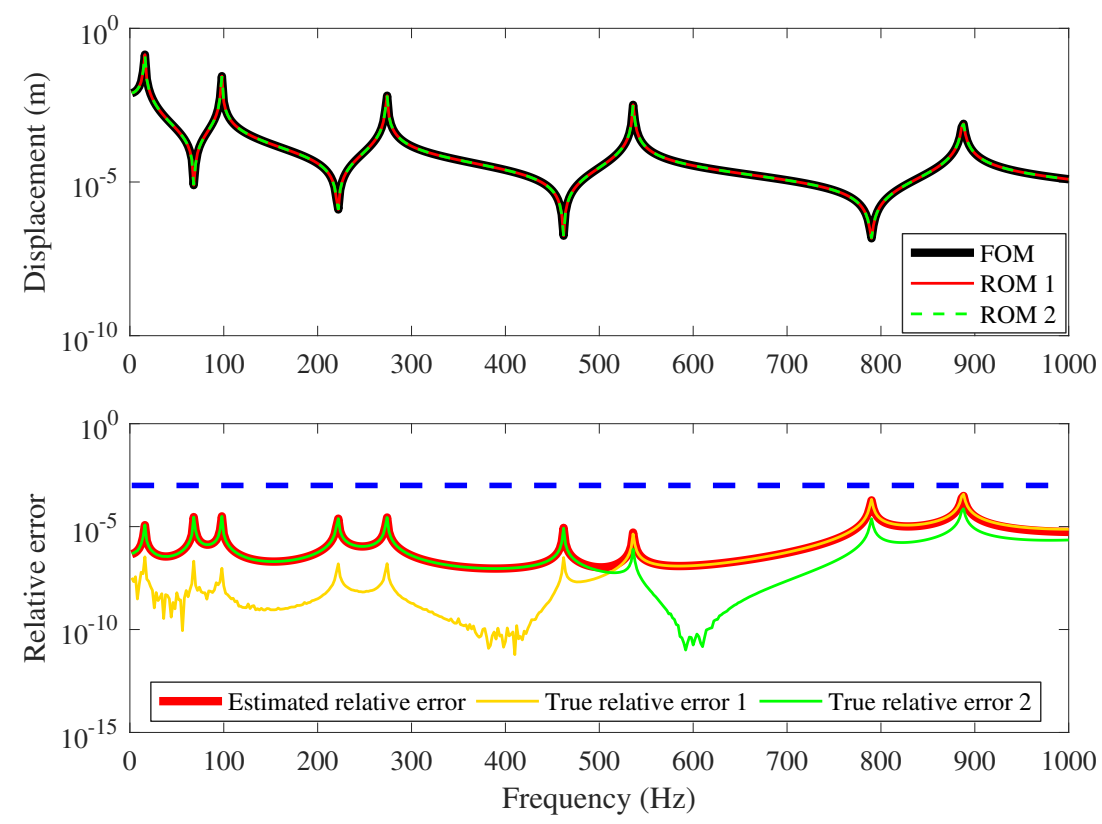

Figure 5: Frequency response and relative error of the FOM and two ROMs of the beam with free-layer damping.

The total elapsed times for obtaining two ROMs transfer function $H_{n}(s)$ (including generating the transformed matrix triplet $(\tilde{\mathbf{K}}, \tilde{\mathbf{D}}, \tilde{\mathbf{M}})$, the orthonormal projection basis $\mathbf{Q}_{n}$ and projection step) and FOM transfer function $H_{N}(s)$ are respectively $t=4.860$ seconds and $t_{f}=620.3$ seconds. Most of the processing time $t_{l}=2.207$ seconds for ROMs is spent on handling the LUdecomposition of the matrix $\tilde{\mathbf{K}}$. The extra computational cost for the convergence of the present relative error is very cheap because only two low-order approximations rather than the original system need to be confronted. The computational benefit of the proposed approach based on SOAR-algorithm can be apparently observed with a speed-up ratio of about 127 times.

\subsection{Free-layer damping of a flat plate with GM model}

In this example, a flat plate of dimensions $0.5 \mathrm{~m} \times 0.35 \mathrm{~m} \times 0.6 \mathrm{~mm}$ with 
a self-adhesive viscoelastic layer of dimensions $0.49 \mathrm{~m} \times 0.34 \mathrm{~m} \times 1.5 \mathrm{~mm}$ is considered, as shown in Fig. 6. The free viscoelastic layer is placed centrally on the plate and its material properties can be obtained from Table 2. The material properties of the steel main structure are: $E=210 \mathrm{GPa}, \rho=7800$ $\mathrm{kg} / \mathrm{m}^{3}, \nu=0.3$. The plate is excited through its clamped boundaries with a uniform normal displacement $w=1 \mathrm{~m}$. Due to the symmetry of the problem, only a quarter of the model needs to be computed. Of course, the proper symmetry boundary conditions have to be applied on the symmetry edges. In this way, using the FEM results in a dynamic system of order $N=15572$. The displacement response to the boundary excitation is simulated in the center of the steel plate, on the untreated side.

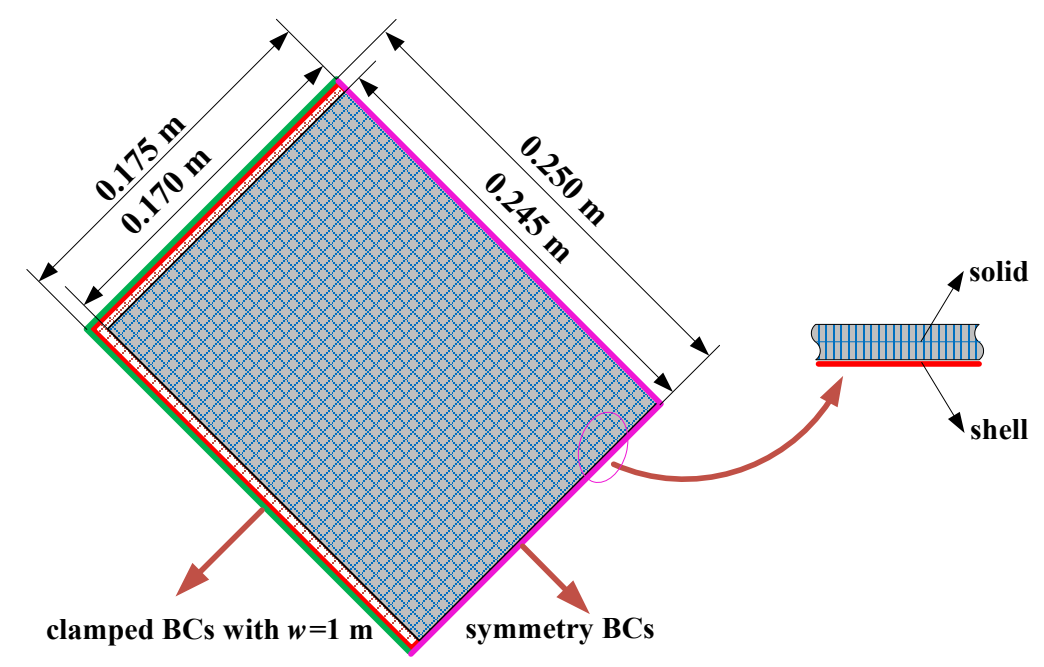

Figure 6: Finite element model of a flat plate with free-layer damping.

The parameter settings for the implementation of SOAR-algorithm are the same as for previous example except for $t o l=1 \mathrm{e}-1$, i.e., $s_{0}^{(1)}=2 \pi \mathrm{j} * 400$, $s_{0}^{(2)}=2 \pi \mathrm{j} * 600, r=p=5$ and $M a x O r d e r=400$. In Fig. 7(a), the frequency responses of the original system and two reduced-order systems are reported. These two ROMs of orders $n=50$ and $n=50$ are terminated for Level 1 since the relative error is less than the pre-specified termination tolerance. The corresponding relative errors are also shown across the frequency range of interest. The estimated relative error clearly indicates that next Level calculation has to be carried out, which is complied with the third case (c) 

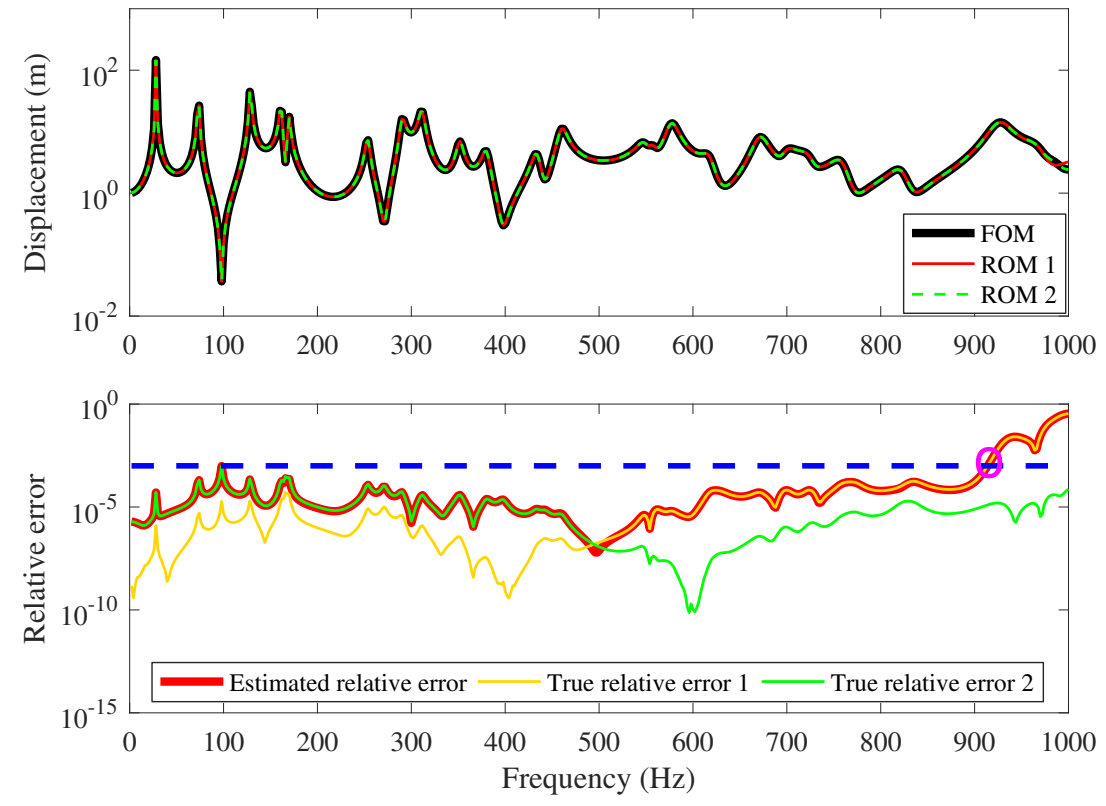

(a)
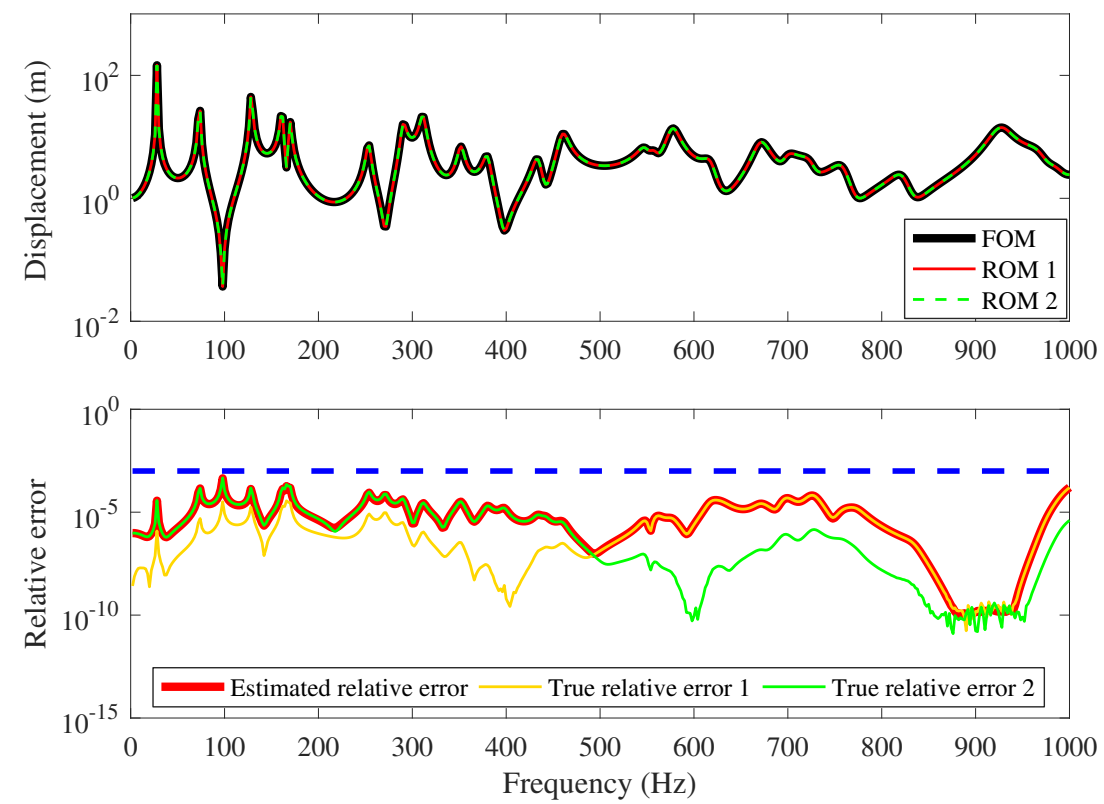

(b)
For ROM 1: $n=50$ New $s_{0}^{(1)}=2 \pi \mathrm{j} * 914$

For ROM 2: $n=50$ New $s_{0}^{(2)}=2 \pi \mathrm{j} * 914$
For ROM 1: $n=60$

For ROM 2: $n=60$

Figure 7: Frequency response and relative error of the FOM and two ROMs of the plate with free-layer damping: (a) Level 1; (b) Level 2. 
of Level 2 in Fig. 2. The values of expansion points that are automatically determined via the proposed error indicator are given along with the figure (see the circle with magenta rim). It should be pointed out that the results of Level 1 for ROM 2 in fact have met the convergence requirements according to the true relative error (see the second figure of Fig. 7(a)), meaning that a large estimated error $\hat{E}(s)$ does not directly imply two large true errors $E(s)$. In other words, where these two different ROMs diverge, maybe only one of them has not yet converged. Keeping in mind however that no information about the true relative error is available a priori, a new expansion point is still needed for ROM 2. After each 10 iterations, we find that two final reduced-order systems of orders $n=60$ and $n=60$ are sufficient for the desired accuracy.

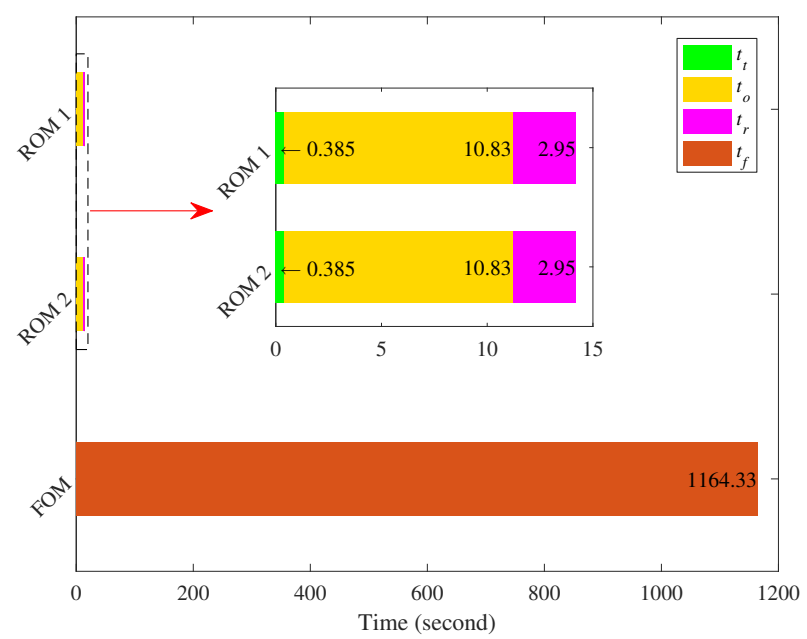

Figure 8: Calculation time for the FOM and various substeps of ROMs.

The elapsed time for obtaining the transfer function of FOM is $t_{f}=$ 1164.33 seconds. The total CPU time needed for two ROMs is $t=28.33 \mathrm{sec}-$ onds. The reduced CPU time gain compared to FOM evaluation is therefore around 41 and thus the speed-up is clear. Specifically, the elapsed times for obtaining the transformed matrix triplet $(\tilde{\mathbf{K}}, \tilde{\mathbf{D}}, \tilde{\mathbf{M}})$, the orthonormal projection basis $\mathbf{Q}_{n}$, the ROM transfer function $H_{n}(s)$ (including the projection step) and the FOM transfer function $H_{N}(s)$ are respectively denoted by: $t_{t}$, $t_{o}, t_{r}, t_{f}$ and presented in Fig. 8. This clearly illustrates that the dominant contribution to the total computational cost of MOR procedure is in the construction of orthonormal basis $\mathbf{Q}_{n}$, of which the LU factorization of $\tilde{\mathbf{K}}$ 
accounts for the largest percentage.

Compared to the previous example, although the order of FOM is slightly smaller, the required CPU time is longer as this is a more complex system, which can be seen from the sparsity patterns of the resulting stiffness matrix $\tilde{\mathbf{K}}$ as shown in Fig. 9. The density of this sparse matrix is 0.00599 for case 1 and 0.00689 for case 2 .
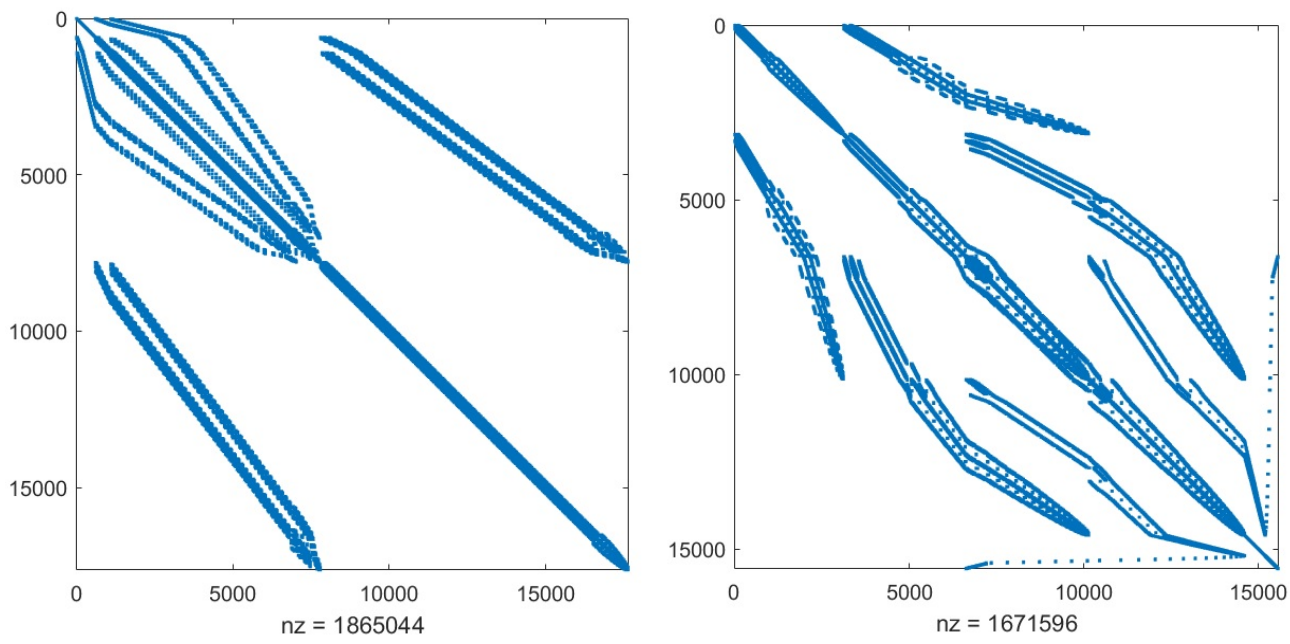

Figure 9: The sparsity pattern of matrix $\tilde{\mathbf{K}}$ : left: case 1 ; right: case 2.

\subsection{Constrained-layer damping of a flat plate with FD model}

In this case, a thin aluminum foil of thickness $0.127 \mathrm{~mm}$ fully covers the viscoelastic material of previous numerical example and forms a sandwich constrained-layer damping system. The viscoelastic material parameters of FD model is in Table 3. Due to the presence of the aluminium top foil, the order of this system is increased to $N=19276$. The parameter settings for the implementation of SOAR-algorithm are chosen as: $s_{0}^{(1)}=2 \pi \mathrm{j} * 400$, $s_{0}^{(2)}=2 \pi \mathrm{j} * 600, r=p=10$, tol $=1 \mathrm{e}-3$ and MaxOrder $=400$. In order to illustrate the versatility of the proposed algorithm, the configured initial parameters are not optimal on the consideration of calculation time and final dimension of the projection basis as well as the ROMs.

Fig. 10(a) illustrates the exact displacement response obtained from the FOM and two SOAR-algorithm estimated displacement responses with the reduced order of $n=80$ and $n=100$. First, compared with the response 


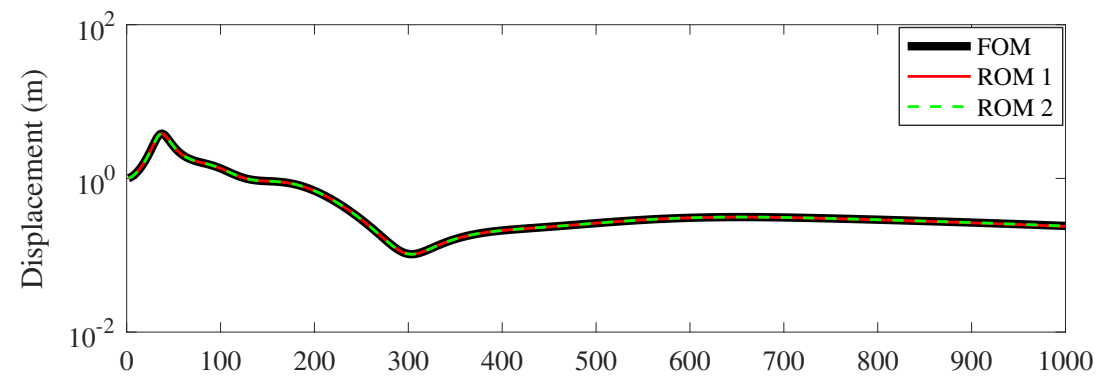

For ROM 1: $n=80$

New $s_{0}^{(1)}=2 \pi \mathrm{j} * 608$

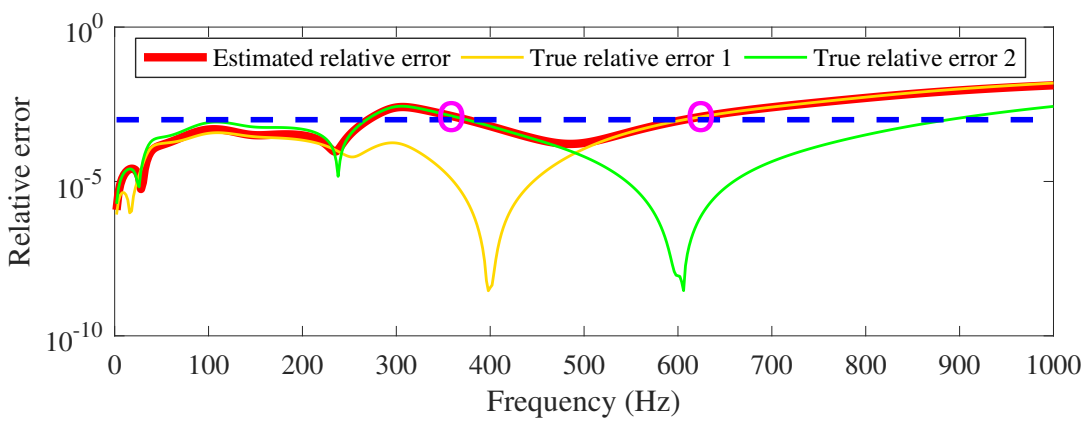

For ROM 2: $n=100$

New $s_{0}^{(2)}=2 \pi \mathrm{j} * 374$

(a)

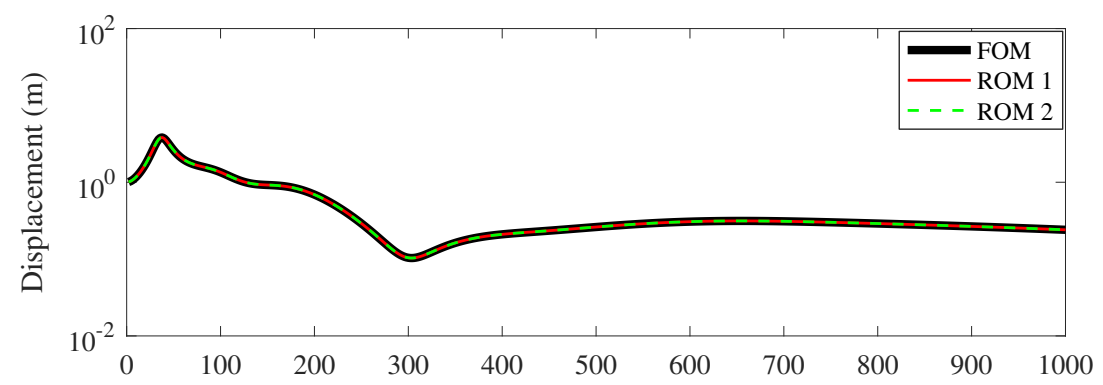

For ROM 1: $n=100$

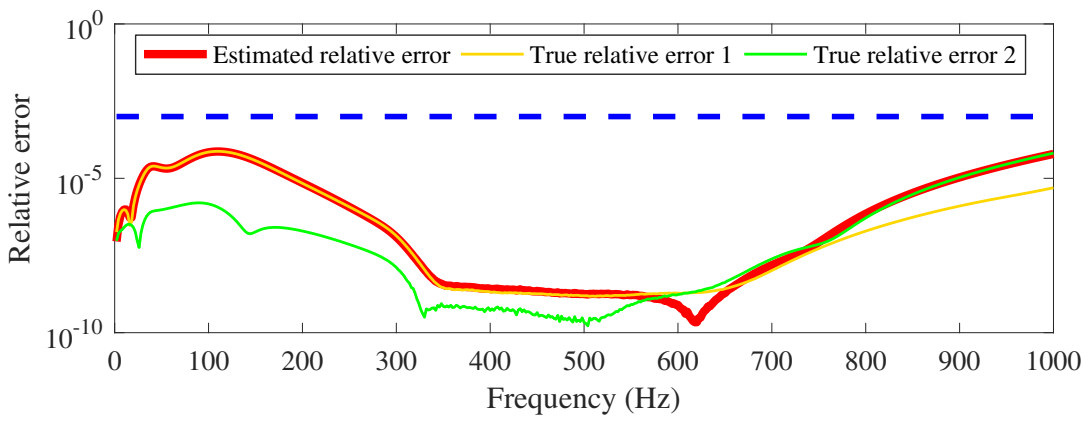

For ROM 2: $n=120$

(b)

Figure 10: Frequency response and relative error of the FOM and two ROMs of the plate with constrained-layer damping: (a) Level 1; (b) Level 2. 
of previous example, it can be intuitively seen that the constrained-layer damping is more effective than the free-layer design since mechanical energy is dissipated more efficiently by the shearing deformation within the viscoelastic layer in comparison to example 2 where there is only bending deformation. Then two intelligently chosen expansion points are also decided (see the circles with magenta rim), which are corresponding to the second case (b) of Level 2 in Fig. 2, i.e, the interleaved expansion points. This result together with the corresponding result from example 2 implies that for both FLD and CLD, multiple expansion points are more accurate than their single expansion point counterpart. This is due to the use of moment information at multiple frequency points, the ROMs satisfy a multi-point moment-matching property and thus reduce the approximation error. Meanwhile, it can be seen from Fig. 10(a) that using only the relative error between two successive reduced models of the order $r$ and $r+p$ is not enough to evaluate the true relative error even if the termination tolerance is taken as tol $=1 \mathrm{e}-3$.

The second plot of Fig. 10(b) shows two true relative errors and their approximation obtained with the present error indicator. According to that figure, finally, two ROMs that can represent the FOM over a wide range of frequency are achieved at $n=100$ and $n=120$, respectively. Obviously, the ROM with $n=100$ should be selected for further application due to the lower dimensions. In spite of the rather flat frequency response of considered structure (see Fig. 10), the order of the ROM required to achieve the desired accuracy is quite high (i.e., $n=100$ ). The reason, on the one hand, is due to the high efficiency of constrained-layer damping. In fact, the modal density is relatively high within the considered frequency range. Also, the term $R_{2}(s) \mathbf{K}_{v}$ in Eq. (10) which is unused to construct the projection subspace contains considerable underlying dynamic information of this system. Therefore, more orthonormal vectors (higher order) are required to enrich the moment-matching property and the projection subspace. On the other hand, it is because that the prescribed initial parameters are not optimal.

In order to investigate the computational accuracy of the proposed methodology, a convergence analysis is performed. The average prediction error $\bar{\varepsilon}$ for the displacement amplitude of response point is defined as:

$$
\bar{\varepsilon}=\frac{1}{N_{\text {freq }}} \sum_{d=1}^{N_{\text {freq }}} \frac{\left|H_{N}^{d}(s)-H_{n}^{d}(s)\right|}{\left|H_{N}^{d}(s)\right|}
$$

where $N_{\text {freq }}$ is the number of discrete frequencies at which the transfer func- 
tion is computed. In this case, $N_{\text {freq }}=500$. Fig. 11 shows the variation of the average error $\bar{\varepsilon}$ with the number of order $n$ (DOFs of the ROM). It is obvious that the error decreases rapidly within the range of $n=10$ to $n=30$ and relatively slowly within the range of $n=40$ to $n=80$ for ROM 1 over the considered frequency range. Furthermore, as a new expansion point is added (at $n=80$ for ROM 1 and at $n=100$ for ROM 2), the prediction accuracy of the proposed approach apparently increases. A similar trend is observed for ROM 2. Again, it can be concluded that for a single expansion point, ROM is impractical to approximate the FOM better by using more orthonormal projection vectors when it reaches a certain value. In such a case, new expansion point has to be added. Although the preferred choice of the reduced order and expansion points changes with the problems to be studied, we have demonstrated that the automatic generation of reduced compact models is feasible by the straightforward implementation of the proposed strategy.

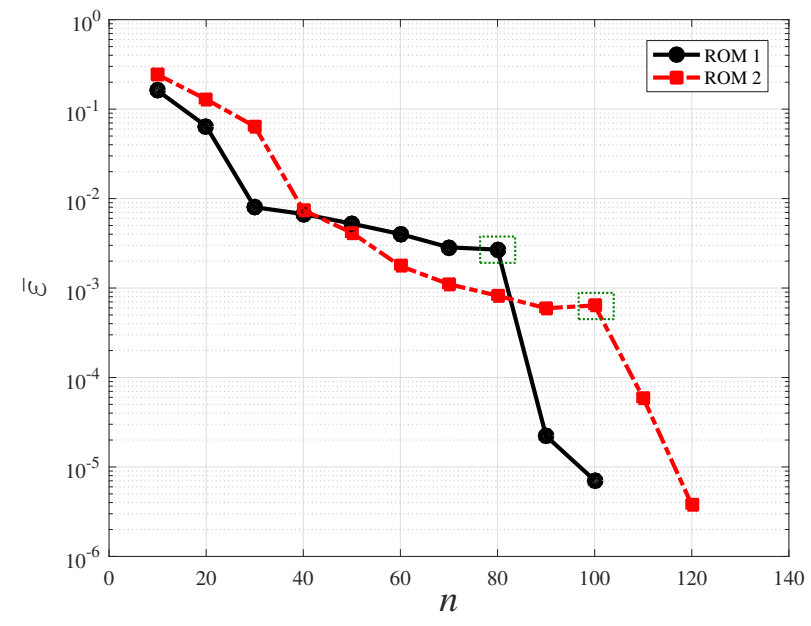

Figure 11: Convergence of ROMs with respect to the order $n$.

In this example, the CPU time required to solve the transfer function for the FOM is $t_{f}=1455.45$ seconds. The total time of the present approach is $t=77.62$ seconds, where the largest proportion of the execution time is undoubtedly used to solve the LU-decomposition. Once again, the CPU time reduction is obvious.

\section{Conclusions}

This paper proposes an efficient strategy for the frequency response anal- 
ysis of complex models consisting of an elastic structure covered by a viscoelastic damping treatment with or without a constrained-layer. Three of the many possible damping models for describing the frequency dependency of the shear modulus are considered to show its versatility. The proposed MOR procedure is based on a local Taylor approximation of the frequency dependent properties of the viscoelastic system and allows to compute a frequency-independent and orthonormal reduction basis that accurately captures the dynamic features of system. This frequency-independent reduction basis is then used in the projection of the full, frequency-dependent system. The Taylor approximation about the selected expansion point is only used to construct this reduction basis, in which SOAR-algorithm based on second-order Krylov subspace is utilized. This way the inherent frequency dependence of the system is preserved in the projection, and is therefore also present in the ROM. As this dependence is typically affine, it is possible to precompute the reduced system matrices, such that the evaluation of the ROM is very cheap and accurate based on proposed relative error indicator. The convergence regarding the order together with the position of expansion points and the number of iterative steps in the final ROM is carefully investigated. We have validated that the presented error indicator provides a cheap tool to ensure sufficient accuracy for the large-scale viscoelastic system, and hence it is a suitable and achievable convergence criterion. The simplicity and high-efficiency of the proposed overall strategy have also been verified.

With this promising strategy, vibro-acoustic simulation can be sped up, especially in the presence of damping materials. In view of efficient parametric studies, it would be worthwhile to further extend present approach to the parametric MOR technique, which allows to retain the parameter dependency in the ROM. Another important extension of this work is that of the inverse characterization of viscoelastic material parameters, which minimize the difference between the numerical representation and the experimental evaluation.

\section{Acknowledgments}

The first author gratefully acknowledges the financial support from China Scholarship Council (CSC NO. 201606230074). The authors X. Xie and H. Zheng thank the National Basic Research Program of China (Grant No. 2014CB046302) for supporting this work. The Research Fund KU Leuven is gratefully acknowledged for its support. The European Commission is 
gratefully acknowledged for their support of the DEMETRA research project (GA 324336).

\section{References}

[1] M. D. Rao, "Recent applications of viscoelastic damping for noise control in automobiles and commercial airplanes," Journal of Sound and Vibration, vol. 262, no. 3, pp. 457-474, 2003.

[2] D. I. G. Jones, "On temperature-frequency analysis of polymer dynamic mechanical behaviour," Journal of Sound and Vibration, vol. 140, no. 1, pp. 85-102, 1990.

[3] S. Jonckheere, Wave Based and Hybrid Methodologies for Vibro-acoustic Simulation with Complex Damping Treatments. PhD thesis, KU Leuven, 2015.

[4] C. W. Bert, "Material damping. An introductory review of mathematic measures and experimental technique," Journal of Sound and Vibration, vol. 29, pp. 129-153, jul 1973.

[5] D. F. Golla and P. C. Hughes, "Dynamics of Viscoelastic Structures-A Time-Domain, Finite Element Formulation," Journal of Applied Mechanics, vol. 52, no. 4, pp. 897-906, 1985.

[6] D. J. McTavish and P. C. Hughes, "Modeling of Linear Viscoelastic Space Structures," Journal of Vibration and Acoustics, vol. 115, no. 1, pp. 103-110, 1993.

[7] S. W. Park, "Analytical modeling of viscoelastic dampers for structural and vibration control," International Journal of Solids and Structures, vol. 38, no. 44-45, pp. 8065-8092, 2001.

[8] R. L. Bagley and P. J. Torvik, "A Theoretical Basis for the Application of Fractional Calculus to Viscoelasticity," Journal of Rheology, vol. 27, no. 3, pp. 201-210, 1983.

[9] T. Pritz, "Analysis of four-parameter fractional derivative model of real solid materials," Journal of Sound and Vibration, vol. 195, pp. 103-115, aug 1996. 
[10] M. Sasso, G. Palmieri, and D. Amodio, "Application of fractional derivative models in linear viscoelastic problems," Mechanics of TimeDependent Materials, vol. 15, pp. 367-387, nov 2011.

[11] J. D. Ferry, Viscoelastic Properties of Polymers. New York: John Wiley \& Sons, 1980.

[12] J. C. Brigham and W. Aquino, "Inverse viscoelastic material characterization using POD reduced-order modeling in acoustic-structure interaction," Computer Methods in Applied Mechanics and Engineering, vol. 198, pp. 893-903, feb 2009.

[13] L. Rouleau, J.-F. Deü, and A. Legay, "A comparison of model reduction techniques based on modal projection for structures with frequency-dependent damping," Mechanical Systems and Signal Processing, vol. 90, pp. 110-125, 2017.

[14] C. Leblond and J. F. Sigrist, "A reduced basis approach for the parametric low frequency response of submerged viscoelastic structures," Finite Elements in Analysis and Design, vol. 119, pp. 15-29, oct 2016.

[15] S. Jonckheere, X. Li, and W. Desmet, "A matrix-free Model Order Reduction scheme for vibro-acoustic problems with complex damping treatments," in Proceedings of the International Conference on Noise and Vibration Engineering, (Leuven, Belgium), sep 2016.

[16] C. Park, D. Inman, and M. Lam, "Model reduction of viscoelastic finite element models," Journal of Sound and Vibration, vol. 219, pp. 619-637, jan 1999.

[17] M. I. Friswell and D. J. Inman, "Reduced-Order Models of Structures with Viscoelastic Components," AIAA Journal, vol. 37, pp. 1318-1325, oct 1999.

[18] S. Zghal, M. L. Bouazizi, N. Bouhaddi, and R. Nasri, "Model reduction methods for viscoelastic sandwich structures in frequency and time domains," Finite Elements in Analysis and Design, vol. 93, pp. 12-29, jan 2015. 
[19] Z. Bai and Y. Su, "SOAR: A second-order Arnoldi method for the solution of the quadratic eigenvalue problem," SIAM Journal on Matrix Analysis and Applications, vol. 26, pp. 640-659, jan 2005.

[20] Z. Bai and Y. Su, "Dimension reduction of large-scale second-order dynamical systems via a second-order Arnoldi method," SIAM Journal on Scientific Computing, vol. 26, pp. 1692-1709, jan 2005.

[21] D. Lu, Y. Su, and Z. Bai, "Stability analysis of the two-level orthogonal Arnoldi procedure," SIAM Journal on Matrix Analysis and Applications, vol. 37, no. 1, pp. 195-214, 2016.

[22] H. K. F. Panzer, Model Order Reduction by Krylov Subspace Methods with Global Error Bounds and Automatic Choice of Parameters. PhD thesis, Technische Universität München, 2014.

[23] Z. Bai and Q. Ye, "Error estimation of the Pad'e approximation of transfer functions via the Lanczos process," Electronic Transactions on Numerical Analysis, vol. 7, pp. 1-17, 1998.

[24] T. Bechtold, E. B. Rudnyi, and J. G. Korvink, "Error indicators for fully automatic extraction of heat-transfer macromodels for MEMS," Journal of Micromechanics and Microengineering, vol. 15, no. 3, pp. 430-440, 2005 .

[25] R. Srinivasan Puri, D. Morrey, A. J. Bell, J. F. Durodola, E. B. Rudnyi, and J. G. Korvink, "Reduced order fully coupled structural-acoustic analysis via implicit moment matching," Applied Mathematical Modelling, vol. 33, pp. 4097-4119, nov 2009.

[26] Q. Xu and H. Zhu, "An inverse model and mathematical solution for inferring viscoelastic properties and dynamic deformations of heterogeneous structures," Computer Methods in Applied Mechanics and Engineering, vol. 300, pp. 798-833, 2016.

[27] T. Pritz, "Five-parameter fractional derivative model for polymeric damping materials," Journal of Sound and Vibration, vol. 265, no. 5, pp. 935-952, 2003. 
[28] Y. Lin, L. Bao, and Y. Wei, "Model-order reduction of large-scale kthorder linear dynamical systems via a kth-order Arnoldi method," International Journal of Computer Mathematics, vol. 87, pp. 435-453, feb 2010 .

[29] T. Koyama and S. Govindjee, "Moment Matching Theorems for Dimension Reduction of Higher-Order Dynamical Systems via Higher-Order Krylov Subspaces," tech. rep., Department of Civil and Environmental Engineering, University of California, Berkeley, 2008.

[30] R. W. Freund, "Recent Advances in Structure-Preserving Model Order Reduction," in Simulation and Verification of Electronic and Biological Systems, pp. 43-70, 2011.

[31] E. J. Grimme, Krylov projection methods for model reduction. PhD thesis, University of Illinois at Urbana-Champaign, 1997.

[32] A. M. G. de Lima, M. H. Stoppa, and D. A. Rade, "Finite Element Modeling of Structures Incorporating Viscoelastic Materials," in Proceedings to XXl IMAC, (Hyatt Orlando, Florida), 2003.

[33] R. Pirk, L. Rouleau, V. D'Ortana, W. Desmet, and B. Pluymers, "Modeling viscoelastic damping insertion in lightweight structures with generalized Maxwell and fractional derivative models," in Proceedings of ISMA2014 Including USD2014, (Leuven, Belgium), pp. 2165-2178, 2014.

[34] L. Rouleau, B. Pluymers, and W. Desmet, "Characterisation of viscoelastic layers in sandwich lightweight panels through inverse techniques," in NOVEM2015, (Dubrovnik, CROATIA), pp. 759-769, 2015.

[35] "COMSOL Multiphysics. https://www.comsol.com/comsolmultiphysics." 